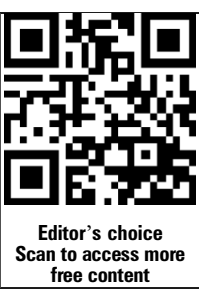

1 Department of Pancreatic Surgery, West China Hospital, Sichuan University, Chengdu, China

${ }^{2}$ Rehabilitation Medicine Center, West China Hospital, Sichuan University, Chengdu, China

\section{Correspondence to} Dr Xubao Liu, Department of Pancreatic Surgery, West China Hospital, Sichuan University, Chengdu 610041, China; hx_xbliu@163.com, xbliu@medmail.com.cn

Received 6 January 2016 Revised 5 February 2016 Accepted 8 February 2016 Published Online First

4 March 2016

\title{
Circular RNAs: a new frontier in the study of human diseases
}

\author{
Yonghua Chen, ${ }^{1}$ Cheng Li, ${ }^{2}$ Chunlu Tan, ${ }^{1}$ Xubao Liu ${ }^{1}$
}

\begin{abstract}
Circular RNAs (circRNAs) are recently discovered new endogenous non-coding RNAs and an area of much research activity. In addition to their potential as major gene regulators, reports are linking heterogeneous circRNA groups with many different human disorders, especially cancer. In this review, we focus on the rapidly advancing field of circRNAs that play a part in human diseases. We list tools (eg, public databases) that scan genome spans of interest to identify known circRNAs; describe the relationship between dysregulated circRNAs and human disease, highlighting their specific roles; and consider the possible use of current and potential circRNA research applications in treating human diseases. Specifically, we review the role of circRNAs as biomarkers, drug targets and therapeutic agents.
\end{abstract}

\section{INTRODUCTION}

Circular RNAs (circRNAs) are a recently discovered new endogenous non-coding RNA (ncRNA) and an area of much research activity. ${ }^{1-3}$ CircRNAs are a large class of RNAs expressed in a tissue-specific and developmental stage-specific manner. ${ }^{1}$ In addition to their potential as a major gene regulator, the heterogeneous group of circRNAs may contribute to the development of many different human disorders. ${ }^{4} 5$ CircRNAs are unusually stable RNA molecules, presumably because their lack of an open end prevents conventional RNA degradation pathways. Thus, circRNAs can indicate gene expression patterns and may be an interesting, new class of biomarkers.

In this review, we focus on circRNAs involved in disease. First, we discuss tools (eg, public databases) that scan genome spans of interest to identify known circRNAs. Some of the databases search for circRNAs that are involved in a specific process or disease (eg, cancer). Second, we present diseaseoriented circRNAs and discuss their relationship with the disease. Finally, we consider their possible clinical implications. Specifically, we review circRNAs as biomarkers, drug targets and therapeutic agents.

\section{CLASSIFICATION OF CIRCRNAS AND PUBLIC DATABASES}

Recently, thousands of human circRNAs were identified using molecular biology strategies coupled with new bioinformatic approaches. Generating comprehensive circRNA classifications is not an easy task. Some circRNAs are only described in one published study. The same circRNA may be listed under different groups, depending on the classification system. For example, circRNAs predicted by computational models are often listed under different names compared with databases obtained from sequencing projects. Our understanding of circRNA biological characteristics and functions is limited; therefore, classifying circRNAs can be achieved only by focusing on their components. The following three types of circRNAs are discussed: intronic circRNAs, exonic circRNAs and exon-intron circRNAs. Intronic circRNAs are produced by connecting two or more introns, which is rare in eukaryotic cells. ${ }^{6}$ Exonic circRNAs are large molecules comprising exons and are considered by-products of exon skipping, either in premessenger RNA (mRNA) splicing or in mature mRNA re-splicing. ${ }^{7}$ The exon-intron circRNAs are enriched at transcription sites and may promote transcription of their parent mRNAs. ${ }^{8} 9$

To enable organisation of circRNAs, we provide the current online databases (table 1). These databases collect circRNAs from GenBank annotations or published articles. They list ncRNAs that have been experimentally proved, those that are purely computational predictions and those annotated as ncRNAs based on the open reading frame predicted size.

Both starBase v2.0 and circBase allow the user to search for functional classes or processes; Circ2Traits ${ }^{10}$ and nc2Cancer ${ }^{11}$ allow the user to search also by disease (eg, cancer). Although the expression datasets are not cancer-oriented, we predict a merging of the circRNA expression databases, listed in table 1 and other datasets that are more disease-oriented (eg, Circ2Traits; http:// gyanxet-beta.com/circdb/). At this time, the genomic positions of several circRNAs can be matched to databases that annotate SNPs associated with disease or disease-associated genetic regions (Circ2Traits ${ }^{10}$ and nc2Cancer $\left.{ }^{11}\right)$. Ghosal et al ${ }^{10}$ measured the likelihood of disease association for a given circRNA from the statistical significance of the interaction of circRNAs with the disease-associated microRNAs (miRNAs). They then analysed gene ontology enrichment on the protein coding genes in the miRNA-circRNA disease interactome to identify enriched genes associated with particular biological processes. Biological process enrichment for mRNAs in 90 diseases was identified. Among these mRNAs, there are 22 light stimulus response genes and 43 cell cycle-related genes associated with breast cancer. ${ }^{10}$ This is the first comprehensive data analysis investigating the global effects of the potential association between circRNAs and cancer.

Recent studies show that miRNAs interact with ncRNAs, such as circRNAs. ${ }^{3} 19$ The circRNAs and mRNAs with common miRNA target sites compete for miRNA binding and form a complex network 
Table 1 Public circRNA databases

\begin{tabular}{|c|c|c|c|c|c|c|c|}
\hline Name & circ2Traits $^{10}$ & nc2Cancer $^{11}$ & $\operatorname{circBase}^{12}$ & starBase v2.0 $0^{13}$ & $\mathrm{CircNet}^{14}$ & deepBase v2.0 $0^{15}$ & Circlnteractome ${ }^{16}$ \\
\hline Website & $\begin{array}{l}\text { http:/l } \\
\text { gyanxet-beta. } \\
\text { com/circdb/ }\end{array}$ & $\begin{array}{l}\text { http://www. } \\
\text { bioinfo.tsinghua. } \\
\text { edu.cn/nc2Cancer }\end{array}$ & $\begin{array}{l}\text { http://www. } \\
\text { circbase.org/ }\end{array}$ & $\begin{array}{l}\text { http://starbase. } \\
\text { sysu.edu.cn }\end{array}$ & $\begin{array}{l}\text { http://circnet.mbc.nctu. } \\
\text { edu.tw/ }\end{array}$ & $\begin{array}{l}\text { http://biocenter. } \\
\text { sysu.edu.cn/ } \\
\text { deepBase/ }\end{array}$ & $\begin{array}{l}\text { http://circinteractome. } \\
\text { nia.nih.gov }\end{array}$ \\
\hline $\begin{array}{l}\text { CircRNA } \\
\text { disease } \\
\text { association }\end{array}$ & 105 Diseases & 31 Cancers & Not available & Not available & Not available & Not available & Not available \\
\hline $\begin{array}{l}\text { CircRNA } \\
\text { annotation }\end{array}$ & $\begin{array}{l}1953 \text { Human } \\
\text { circRNAs }\end{array}$ & $\begin{array}{l}172 \text { Human } \\
\text { circRNAs }\end{array}$ & Not available & Not available & 212950 CircRNAs & $\begin{array}{l}14867 \text { Human } \\
\text { circRNAs }\end{array}$ & Not available \\
\hline $\begin{array}{l}\text { Sequence } \\
\text { alignment }\end{array}$ & Not available & Not available & $\begin{array}{l}\text { A web interface } \\
\text { of BLAST }\end{array}$ & Not available & $\begin{array}{l}\text { A web interface of } \\
\text { BLAST }\end{array}$ & Not available & Not available \\
\hline $\begin{array}{l}\text { CircRNA } \\
\text { reference } \\
\text { source }\end{array}$ & $\begin{array}{l}\text { circRNA dataset } \\
\text { from Memczak } \\
\text { et al }\end{array}$ & $\begin{array}{l}\text { miR2Disease, } \\
\text { miRCancer, } \\
\text { HMDD v2.0 and } \\
\text { starBase }\end{array}$ & $\begin{array}{l}\text { Back-spliced } \\
\text { junction sites in } \\
\text { animals reported } \\
\text { in } 2013 \text { and } \\
2015\end{array}$ & circBase v0.1 & $\begin{array}{l}\text { Reported human } \\
\text { circRNAs from } 2013 \text { to } \\
2015\end{array}$ & $\begin{array}{l}\text { NCBI GEO and } \\
\text { SRA databases }\end{array}$ & $\begin{array}{l}\text { CircBase, starBase } \\
\text { V.2.0, miRBase, }{ }^{17} \\
\text { IRESite }^{18}\end{array}$ \\
\hline $\begin{array}{l}\text { CircRNA } \\
\text { position on } \\
\text { genome }\end{array}$ & $\begin{array}{l}\text { A customised } \\
\text { genome browser }\end{array}$ & Not available & $\begin{array}{l}\text { Linked out to } \\
\text { UCSC Genome } \\
\text { Browser }\end{array}$ & $\begin{array}{l}\text { A customised } \\
\text { genome browser } \\
\text { accessible } \\
\text { through keyword } \\
\text { search }\end{array}$ & $\begin{array}{l}\text { An integrated genome } \\
\text { browser synchronising } \\
\text { with the network } \\
\text { graphical user interface }\end{array}$ & $\begin{array}{l}\text { A customised } \\
\text { genome browser }\end{array}$ & $\begin{array}{l}\text { Linked out to UCSC } \\
\text { genome browser }\end{array}$ \\
\hline $\begin{array}{l}\text { CircRNA sample } \\
\text { source }\end{array}$ & Not available & Not available & $\begin{array}{l}\text { The samples } \\
\text { where the } \\
\text { back-spliced } \\
\text { junction sites } \\
\text { were discovered }\end{array}$ & $\begin{array}{l}\text { circBase v0.1 } \\
\text { source samples }\end{array}$ & $\begin{array}{l}\text { 1. In which sample } \\
\text { junction sites were } \\
\text { discovered. } 2 \text {. Expression } \\
\text { level in available } \\
\text { samples. } 3 \text {. Clustered } \\
\text { sample conditions }\end{array}$ & Not available & Not available \\
\hline $\begin{array}{l}\text { CircRNA } \\
\text { naming }\end{array}$ & $\begin{array}{l}\text { A serial number } \\
\text { for every } \\
\text { detected } \\
\text { back-spliced } \\
\text { junction site }\end{array}$ & $\begin{array}{l}\text { A serial number } \\
\text { for every detected } \\
\text { back-spliced } \\
\text { junction site }\end{array}$ & $\begin{array}{l}\text { A serial number } \\
\text { for every } \\
\text { detected } \\
\text { back-spliced } \\
\text { junction site }\end{array}$ & $\begin{array}{l}\text { Same as circBase, } \\
\text { except CDR1 } \\
\text { antisense } \\
\text { (CDR1as) }\end{array}$ & $\begin{array}{l}\text { A systematic naming } \\
\text { system which provides } \\
\text { information to the } \\
\text { source gene and } \\
\text { annotated exons of } \\
\text { circRNAs }\end{array}$ & $\begin{array}{l}\text { A systematic } \\
\text { naming system } \\
\text { which provides } \\
\text { information to the } \\
\text { transcript number }\end{array}$ & Same as circBase \\
\hline $\begin{array}{l}\text { CircRNA } \\
\text { expression } \\
\text { profiles in } \\
\text { samples }\end{array}$ & Not available & Not available & Not available & Not available & $\begin{array}{l}\text { An all-sample expression } \\
\text { heat-map for every } \\
\text { circRNA and linear } \\
\text { isoform }\end{array}$ & Not available & Not available \\
\hline $\begin{array}{l}\text { Address on } \\
\text { miRNA } \\
\text { regulatory } \\
\text { relationships }\end{array}$ & $\begin{array}{l}\text { Identifies circRNA } \\
\text { and miRNA } \\
\text { interactions }\end{array}$ & $\begin{array}{l}\text { Identifies circRNA } \\
\text { and miRNA } \\
\text { interactions }\end{array}$ & Not available & $\begin{array}{l}\text { Identifies circRNA } \\
\text { and miRNA } \\
\text { interactions } \\
\text { through Chip-Seq } \\
\text { data analysis }\end{array}$ & $\begin{array}{l}\text { A network-driven } \\
\text { graphical interface } \\
\text { shows the relationship } \\
\text { between miRNA target } \\
\text { genes and circRNAs }\end{array}$ & Not available & $\begin{array}{l}\text { Identifies circRNA } \\
\text { and miRNA } \\
\text { interactions }\end{array}$ \\
\hline $\begin{array}{l}\text { RNA-binding } \\
\text { protein }\end{array}$ & Not available & Not available & Not available & Not available & Not available & Not available & $\begin{array}{l}\text { Identifies } \\
\text { RNA-binding protein }\end{array}$ \\
\hline $\begin{array}{l}\text { circRNA } \\
\text { isoforms }\end{array}$ & Not available & Not available & Not available & Not available & $\begin{array}{l}\text { All traceable on the } \\
\text { integrated genome } \\
\text { browser }\end{array}$ & $\begin{array}{l}\text { All traceable on } \\
\text { the integrated } \\
\text { genome browser }\end{array}$ & Not available \\
\hline
\end{tabular}

of interaction and regulation, commonly known as the competing endogenous RNA (ceRNA) network. Dysregulated crosstalk between ceRNAs in the network plays an important role in cancer pathogenesis. Mutations in miRNAs (especially in seed regions) and their target sites may alter miRNA-ceRNA interactions and rewire the ceRNA network. The SomamiR 2.0 database contains somatic mutations in miRNAs and their target sites for three types of ceRNAs-circRNAs, long ncRNAs and miRNAs. $^{20}$

\section{CIRCRNAS IN HUMAN DISEASES}

CircRNAs participate in a wide range of biological processes. Almost every step in a gene's life cycle, including transcription, mRNA splicing, RNA decay and translation, can be influenced by circRNAs. ${ }^{21}$ Considering the wide range of roles that circRNAs play in cellular networks, it is not surprising that their misregulation leads to abnormal cellular functions and growth defects and is implicated in human disease. We focused on developing a list of circRNAs linked to human disease by various means. We mainly used two of the online databases to retrieve circRNAs (Circ2 Traits $^{10}$ and nc2Cancer ${ }^{11}$ databases) and we searched PubMed for articles linking these circRNAs to human disease. In table 2, we summarise our findings.

Several circRNAs are causally involved in human disease. In some cases, the link between the circRNA and human disease was obvious and human disease was the model in which these circRNAs were first described (eg, the exonic circRNAs that are antisense cerebellar degeneration-related protein 1 transcript (CDR1as) and sex-determining region Y (Sry)). However, we also found some circRNAs with incompletely elucidated links to human disease, but preliminary findings suggest that further investigation of possible connections is needed. Here, we focus on the genetic and epigenetic events that disrupt circRNA loci and related proteins in cancer and other human diseases, such 
Table 2 CircRNAs associated with human disease

\begin{tabular}{|c|c|c|c|c|c|c|}
\hline CircRNA & Alias & Regulation & Gene symbol & Sample & Disease/tumour & Genome position \\
\hline hsa_circ_0001946 & CDR1as & - & CDR1 & Cell lines & Colorectal cancer, ${ }^{22} 23$ breast cancer ${ }^{22} 24$ & chrX:139865339-139866824 \\
\hline hsa_circ_0001946 & CDR1as & $\downarrow$ & CDR1 & Human samples & Alzheimer's disease, ${ }_{i}^{25}$ & chrX:139865339-139866824 \\
\hline hsa_circ_0001141 & hsa_circ_001763 & $\downarrow$ & $\mathrm{ITCH}$ & Human samples & Colorectal cancer, ${ }^{26}$ oesophageal carcinoma ${ }^{27}$ & chr20:33001547-33037285 \\
\hline hsa_circ_0006229 & - & $\downarrow$ & TNS3 & Human samples & Colorectal cancer $^{28}$ & chr7:47384352-47385954 \\
\hline hsa_circ_0007374 & - & $\uparrow$ & AZIN1 & Human samples & Colorectal cancer ${ }^{28}$ & chr8:103846416-103852051 \\
\hline hsa_circ_0002190 & circ-KLDHC10 & $\uparrow$ & KLDHC10 & $\begin{array}{l}\text { Human samples } \\
\text { (cancer serum) }\end{array}$ & 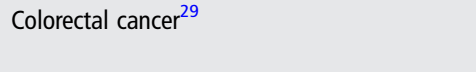 & chr7:129760588-129762042 \\
\hline hsa_circ_0001649 & hsa_circ_001599 & $\downarrow$ & SHPRH & Human samples & Hepatocellular carcinoma ${ }^{30}$ & chr6:146209155-146216113 \\
\hline hsa_circ_0000140 & hsa_circ_0002059 & $\downarrow$ & KIAA0907 & Human samples & Gastric $_{\text {cancer }}{ }^{31}$ & chr1:155891165-155895634 \\
\hline hsa_circ_0100783 & - & $\uparrow$ & PCDH9 & Human samples & Immunosenescence $\mathrm{e}^{32}$ & chr13:67750008-67750243 \\
\hline
\end{tabular}

as neurological, cardiovascular and autoimmune disorders. We next illustrate several circRNAs and their involvement in human diseases.

\section{CircRNAs: deregulation in cancer}

In cancer biology, the search for gene expression differences between tumour and normal samples is considered most important, as knowledge of circRNA expression profiles in tumour and normal samples is modest. Commercial gene expression arrays used for polycomb group proteins may contain probes that hybridise to circRNAs and it may be possible to retrieve cancerrelated circRNA expression profiles from public, tumour-specific, gene-expression datasets (eg, Circ2Traits and nc2Cancer). To identify new transcripts, some investigators have used the Arraystar Human CircRNA array, which can test for circRNA gene expression. ${ }^{33}$ Through the microarray platform, Qu et al ${ }^{33}$ identified dysregulated circRNA expression signatures of pancreatic carcinoma. Others have performed custom array profiling on large sample sets of a few circRNAs. Most articles of circRNA expression in cancer examine a select number of circRNAs in tumour samples. Cancer biologists sought to uncover genetic mutations in circRNA sequences. For example, researchers found that circRNAs are globally reduced in colorectal cancer (CRC) tissues via RNA sequencing data analysis of 12 matched normal colon mucosa and tumour tissues. ${ }^{28}$ Analysis of circRNA expression profiles (and other ncRNAs) in a variety of cancer patient samples in comparison with the corresponding normal cells, demonstrated dysregulation in a wide range of cancers. We outline the roles of various circRNAs in different types of cancer.

\section{Abnormal expression of circRNAs in cancers}

Many circRNAs show tissue-specific and developmental stagespecific expression patterns ${ }^{1}$ and play critical roles in cancerrelated biological processes. Several circRNAs are essential for attaining and maintaining cancer phenotypes and are dysregulated in a wide range of cancers.

A recent study found that the global abundance of circRNAs was lower in CRC than in normal tissue. ${ }^{28}$ Remarkably, Li et $a l^{27}$ have shown that cir-ITCH (also known as hsa_circ_0001141 or hsa_circ_001763) expression is typically downregulated in oesophageal squamous cell carcinoma in comparison with paired adjacent tissue. In addition, Huang et $a l^{26}$ drew the similar conclusion that cir-ITCH expression is typically downregulated in CRC in comparison with peritumoral tissue. In gastric cancer, researchers have discovered that hsa_circ_002059 is downregulated. ${ }^{31}$ Qin et al ${ }^{30}$ showed that hsa_circ_0001649 is downregulated in hepatocellular carcinoma (HC̄C) via qRT-PCR.
Li et $a l^{29}$ examined serum exosome (small membrane vesicles of endocytic origins secreted by cells) RNA sequencing datasets from 11 patients with CRC and normal serum. In comparison with healthy subjects, 67 circRNAs were missing and 257 new circRNA species were detected in the patients with CRC. Notably, many of the host genes for these new circRNAs (48 genes for 53 circRNAs) were significantly upregulated in CRC tissues. qRT-PCR analysis confirmed that the expression levels of circ-KLDHC10 (also known as hsa_circ_0002190) were upregulated in CRC serum. ${ }^{29}$

In addition, a recent review reported that expression analyses of various tumour cell lines showed widespread expression of ciRS-7 in neuroblastomas, astrocytoma, renal cell and lung carcinomas. $^{22}$ The stable expression of ciRS-7 in HeLa cells ${ }^{19}$ indicates that ciRS-7 may be associated with cervical cancer.

CircRNAs are differentially expressed in a wide range of cancers and may be involved cancer initiation and progression. Continuing research into circRNAs shows that they may play roles in other tumours and are potential new biomarkers for diagnosis. For example, seral exosomal circRNAs profiles can distinguish between patients with cancer and healthy controls. ${ }^{29}$

\section{CircRNAs are associated with cancer-related miRNAs}

It is clear that miRNAs are involved in nearly all aspects of cellular functions ${ }^{34}$ and have a critical role in cancer initiation and progression. ${ }^{35} 36$ In March 2013, two publications reported that circRNAs function as miRNA 'sponges', which naturally sequester and competitively suppress the activity of miRNAs. ${ }^{1} 19$ The circRNAs and mRNAs with common miRNA target sites compete for miRNA binding and form a complex interactive and regulatory network, commonly known as the ceRNA network. The miRNA target recognition is largely dependent on sequence complementarity between the miRNA seed region (nucleotides 2-7 in the mature miRNA sequence) and its target sites on ceRNAs. Mutations in miRNAs (especially the seed regions) and their target sites may alter miRNA-ceRNA interactions and rewire the ceRNA network. ${ }^{37}$ The dysregulation of crosstalk between ceRNAs in the network has an important role in cancer pathogenesis, suggesting that circRNAs might be involved in malignant tumours correlated with miRNAs.

By measuring the likelihood of a circRNA association with disease from the statistical significance of their interaction with miRNAs associated with the disease in question, the Circ2Traits database $^{10}$ listed biological processes for mRNAs in 90 diseases. Among these mRNAs, there are 22 light stimulus response genes and 43 cell cycle-related genes associated with breast cancer. ${ }^{10}$ This is the first report with a global view of the potential association between circRNAs and cancer based on comprehensive 
data analysis. However, a direct circRNA and miRNA association requires more biological evidence. The SomamiR 2.0 database contains somatic mutations in miRNAs and their target sites on circRNAs, long ncRNAs and miRNAs. ${ }^{20}$

Identifying ceRNAs and circRNAs as important regulators of miRNA activity underlines the increasing complexity of ncRNA-mediated regulatory networks. In particular, the recently identified circRNA, known as ciRS-7, which acts as a ceRNA or super sponge of miR-7, competitively inhibits miR-7 activity and promotes oncogene expression (such as EGFR and XIAP), while inhibiting tumour suppression genes (such as KLF4) and therefore promoting the initiation and development of cancer, such as HCC, breast cancer and cervical cancer. ${ }^{5} 22$ Hence, discovering the regulation of miR-7/miR-671/ciRS-7 axis activity will probably advance the understanding of various cancer aetiologies. ${ }^{5}$ Another notable circular miRNA sponge is Sry. Sry controls the biological effects of miR-138 by binding to its 16 conserved binding sites. ${ }^{19}$ It can regulate complex regulatory networks and influence many physiological and pathological processes, by modulating multiple miRNAs. Because both Sry and miR-7 have a crucial effect on the occurrence and progression of cancer, we assume that circRNAs must also be involved in the process.

\section{CircRNA-miRNA axes regulate cancer-related pathways}

Some endogenous circRNAs in mammalian cells are highly abundant and evolutionarily conserved in oncogenesis. To date, the evidence suggests that circRNAs may regulate transcription and pathways by manipulating miRNAs. The ciRS-7/miR-7 axis is probably involved in cancer-associated biological processes, such as cancer initiation and progression. ${ }^{5} 22$ The circRNA regulatory influence on miR-7 is clear; ciRS-7 overexpression acts as a miRNA sponge to restrain the expression miR-7 and therefore elevates EGFR expression. ciRS-7 can naturally sequester and inhibit miR-7 activity and promote oncogenic EGFR and XIAP gene expression as well as inhibit the tumour-suppressor KLF4 expression, thus promoting the initiation and development of cancer, such as cervical cancer, breast cancer and HCC. ${ }^{5} 22$ Additionally, miR-7 indirectly upregulates E-cadherin by targeting FAK $^{24}$ and IGF1R, ${ }^{38}$ resulting in reduced epithelial to mesenchymal transition (EMT), decreased anchorage-independent growth and suppression of metastasis. ${ }^{4}$

Li $e a l^{27}$ have shown that cir-ITCH may have an antitumour function in oesophageal squamous cell carcinoma through interactions with miRNAs such as miR-7, miR-17 and miR-214 and an increase in ITCH. These interactions facilitate ubiquitinmediated Dvl2 degradation and decrease expression of the oncogene c-myc. This process therefore inhibits canonical Wnt signalling. In addition, Huang et $a l^{26}$ drew the parallel conclusion that cir-ITCH expression is typically downregulated in $\mathrm{CRC}$, and that cir-ITCH has an inhibitory role in the canonical Wnt pathway, inhibiting c-myc and cyclinD1 expression.

A recent study indicated that the majority of circRNAs are regulated by human EMT and more than one-third of abundant circRNAs are dynamically regulated by the alternative splicing factor, Quaking, which is regulated by the EMT process. ${ }^{39}$ Given that EMT participates in tumorigenesis and provides important indications for cancer progression, we may obtain more knowledge of the therapeutic role of circRNA by targeting miRNAs involved in EMT.

\section{CircRNAs involved in neurological disorder}

CircRNAs play a critical role in normal nervous system function and during various differentiation stages. Most circRNA expression is associated with specific neuroanatomical regions, cell types or subcellular compartments. Moreover, circRNA levels increase relative to linear mRNAs during ageing. ${ }^{40} 41$ Recently, two papers by Rybak-Wolf et $a l^{42}$ and You et $a l^{43}$ provide a valuable circRNA catalogue of the mammalian brain and shed a new light on their potential function in the nervous system. The authors ${ }^{42}{ }^{43}$ report the identification of thousands of conserved circRNAs that are highly expressed in mammalian brain. Many of these circRNAs are upregulated during neurogenesis and further enriched in synaptic processes compared with their linear isoforms. These findings further highlight a potential role of brain circRNAs in the nervous system. Rybak-Wolf $e t a l^{42}$ examined 29 human/mouse RNA sequencing datasets from dissected brain tissues or neuronal-differentiated cell lines. They found that some circRNAs are expressed dynamically and independently of their linear transcripts, implying regulated expression of brain circRNAs. You et $a l^{43}$ drew the parallel conclusion that circRNAs are highly enriched and developmentally regulated in the brain. Therefore, circRNA dysregulation causes various neurodegenerative and neurological disorders. CircRNAs have been implicated in neurological disorders, such as Alzheimer's disease (AD), ${ }^{25}$ Parkinson's disease ${ }^{10}$ and many others. We review the association of various circRNAs with neurological disorders.

The discovery of CDR1as, a miR-7 sponge, promoted theories about the link between circRNAs and neurodegenerative diseases such as AD, since miR-7 and some other microRNAs are directly related to neurodegenerative diseases. ${ }^{25}$ For example, CDR1as is highly expressed in the brain and has over 60 binding sites for miR-7. ${ }^{19}{ }^{44}$ It is important to note that miR-7 is implicated in numerous pathways and diseases, including its function as a direct regulator of $\alpha$-synuclein and ubiquitin protein ligase A. CDR1 as has been implicated in Parkinson's disease, ${ }^{10} \mathrm{AD}^{25}$ and brain development. ${ }^{19}$ A recent report supports the downregulation of CDR1as in patients with Alzheimer's disease. ${ }^{25}$ Using northern blot hybridisation techniques and the circularitysensitive circRNA probe, RNaseR, Lukiw ${ }^{25}$ provided evidence of a misregulated miR-7-circRNA system in the sporadic AD hippocampal CA1 region. The author found that deficits in ciRS-7 and ciRS-7 'sponging activities' increase ambient miR-7 levels in AD-affected brain cells, ultimately downregulating selective miR-7 targets. Upregulated miR-7, due to a deficiency in ciRS-7 'sponging' effects, is predicted to downregulate AD-relevant targets, such as the ubiquitin protein ligase $\mathrm{A}$ (UBE2A). UBE2A, which is depleted in AD brains, is an autophagic, phagocytic protein that is essential for the clearance of amyloid peptides in $\mathrm{AD}$ and other progressive inflammatory degenerations of the human central nervous system.

Amyotrophic lateral sclerosis is a devastating neurodegenerative disease, primarily affecting motor neurons. Mutations in the gene encoding TDP-43 cause some forms of the disease, and cytoplasmic TDP-43 aggregates accumulate in degenerating neurons of most people with amyotrophic lateral sclerosis. Thus, strategies targeting the toxicity of cytoplasmic TDP-43 aggregates may be effective. A study by Armakola $e t a l^{45}$ shows that in the absence of Dbr1 enzymatic activity, intronic lariats accumulate in the cytoplasm to sequester TDP-43, preventing the aggregates from interfering with essential cellular RNAs and RNA-binding proteins. Dbr1 knockdown in a human neuronal cell line or in primary rat neurons is sufficient to suppress TDP-43 toxicity. ${ }^{45}$

These studies suggest that circRNAs have a crucial role in neurological functions, although their mechanisms are unknown. The specific expression and stability of circRNAs 
make them interesting candidates as biomarkers for neurodegenerative diseases, such as $\mathrm{AD}$.

\section{Disruption of circRNAs in other diseases}

CircRNAs are disrupted and associated with cardiovascular disorders. For atherosclerotic vascular disease (ASVD), Burd et al ${ }^{46}$ discovered that expression of the new circRNA transcript circular antisense non-coding RNA in the INK4 locus (cANRIL) may be correlated with INK4/ARF transcription and ASVD risk. Based on genome-wide association study studies, SNPs in noncoding regions have been shown to be associated with higher susceptibility to a range of diseases. A large number of SNPs in the INK4/ARF loci were associated with increased ASVD risk. ${ }^{47} 48$ The chromosome region in which the SNPs were characterised harbours the protein-coding genes CDKN2a (INK4a) and CDKN2b (INK4b). Both these genes are adjacent to the gene encoding cANRIL. cANRIL is an antisense transcript from the INK4A/ARF locus. ${ }^{49}$ SNPs on chromosome 9p21.3 near the INK4/ARF (CDKN2a/b) locus within the ASVD risk interval may modulate ANRIL splicing and cANRIL production. ${ }^{46}$ Hence, cANRIL could be involved in ASVD.

Ashwal-Fluss $e t a l^{50}$ discovered that a new circRNA transcript called CircMbl might be correlated with myotonic dystrophy initiation and progression. CircMbl and its flanking intron sequences can combine with MBL. Alterations in MBL levels strongly affect circMbl biosynthesis. CircRNA production competes with canonical mbl pre-mRNA splicing. ${ }^{50} \mathrm{MBL}$ can regulate mbl pre-mRNA splicing efficiency through the activity of both mbl mRNA and circMbl. Moreover, circMbl can act as an MBL sponge. MBL functional deficiency causes a severe degenerative disease called myotonic dystrophy. Hence, circMbl could be involved in myotonic dystrophy initiation and progression.

Aberrant immune responses are a hallmark of ageing and age-associated diseases, associated, in part, with the continuous proinflammatory cytokine secretion by senescent cells. Recent findings link circRNAs to CD28-related CD8(+) T cell ageing and global immunosenescence. Using a cross-comparison of circRNA microarrays and stepwise bioinformatic assays, Wang et $a l^{32}$ investigated circular RNA-micro RNA interactions in ageing human $\mathrm{CD} 8(+) \mathrm{T}$ cell populations and examined the accompanying loss of CD28 expression. The authors discovered that circRNA100783 might regulate phosphoprotein-related signal transduction during CD28-dependent $\mathrm{CD} 8(+) \mathrm{T}$ cell ageing.

The current circRNA field is based upon disease-associated changes in circRNA expression. However, genetic studies on circRNA sequences may distinguish the specific contribution of large- and small-scale mutations to circRNA functions. With improved understanding of circRNA language, we will be able to classify diseases based on the mutations identified and their effect on circRNA function.

\section{DIAGNOSTIC OR THERAPEUTIC APPLICATION OF CIRCRNAS}

The relatively new field of circRNA research is expanding quickly, but many gaps remain. Only recently has the circRNA number in the human genome become clear. Researchers have not extensively investigated circRNA expression in large, clinically controlled tumour datasets, and circRNA functions are not well understood. Few examples of transgenic circRNA models have been published. Recent work suggested that circRNAs may have an important role in the initiation and development of disease. We foresee a potential use for circRNAs in the clinical setting.

\section{Diagnostic and prognostic biomarkers}

According to the current literature, the main characteristics of circRNAs are as follows: ${ }^{51} 52$ (i) universality; (ii) conservatism, as the signal behind circularisation seems to be evolutionarily conserved among different species; ${ }^{21}$ (iii) definite specificity; ${ }^{52}$ (iv) stability, or a resistance to debranching enzymes and RNA exonucleases and (v) highly abundant expression, as the gene product level of some exonic circRNAs is higher than that of linear RNAs. ${ }^{53}$ Therefore, circRNAs possess distinct advantages and may be useful as new biomarkers for diagnosis, prognosis and therapeutic response prediction. ${ }^{29}$

The marked increase or decrease in circRNA expression levels in tumours compared with normal tissues seems to be a feature shared by circRNAs that would be useful in diagnostics. There are a few examples of circRNAs with a diagnostic role. For example, several circRNAs are reportedly aberrantly expressed in human cancers (such as, oesophageal carcinoma, ${ }^{27}$ $\mathrm{CRC}^{28}$ and gastric cancer ${ }^{31}$ ). Upregulation or downregulation of circRNAs in cancer tissues compared with paired adjacent tissue could indicate a diagnostic potential new biomarker. A large difference in circRNA tumour expression levels compared with normal tissues is a topic for future clinical research, although larger clinical datasets need to be assayed. Other circRNAs might also be promising biomarkers. ${ }^{29}$ CircRNAs have been described as an ageing biomarker class in Drosophila. ${ }^{40}$

A potential avenue of circRNA research is the circulating circRNAs in serum, plasma ${ }^{54}$ and other body fluids, ${ }^{55} 56$ especially in microparticles, like exosomes. ${ }^{29}$ One of the main advantages of circRNAs is their high circulating stability. They can be detected through minimally invasive blood, urine or saliva sample collection coupled with RT-PCR. ${ }^{54-56}$ This might represent an unexpected and unexplored potential disease biomarker for diagnosis, prognosis and therapeutic response prediction. $^{55} 56$

\section{Contribution to targeted therapy}

CircRNAs might be useful therapeutic agents. Circularisation may be a future target for treatment, either to reduce the circularisation of functional transcripts or to use an 'mRNA trap' to sequester dysfunctional exons in transcripts. ${ }^{3} 22$ Circularising the miRNA sponge in cells is a new candidate for RNA-based cancer treatments. ${ }^{57}$ Researchers recently reported that a circular, artificial, miRNA sponge might induce miRNA loss-of-function in cancer cells. ${ }^{57}$ Liu et al ${ }^{57}$ constructed a circular miRNA sponge expression vector containing the permuted intron-exon sequence derived from the group I intron of T4 bacteriophage gene td and produced circular miRNA sponges against miR-21 and miR-221. As alternative vectors expressing linear sponges, the expression vectors for RNA circles described in this study open new ways to deliver miRNA sponges with persistent effects and hold great potential for cancer research and treatment. Endogenous circRNAs were recently identified as a new class of gene expression regulators, acting as miRNA sponges in mammals. ciRS-7 functions as a potent circular miR-7 sponge, containing multiple miRNA response elements that bind miR-7. ${ }^{58}$ Thus, it can instantaneously bind or release a large number of miR-7 molecules, thereby effectively regulating the disease network. ${ }^{5}$ The miRNA sponge function of endogenous circRNAs is a general phenomenon. Studying the evolutionarily optimised, circular miRNA sponge structures should provide valuable insights into the design and development of potent artificial sponges in order to achieve effective inhibition of miRNA function in diseases. 
ncRNAs broaden the universe of potential 'druggable' targets. The scientific community and pharmaceutical companies must pursue these new approaches vigorously, using automated large-scale screening of these miRNA-related drugs, developing knock-in and knockout models for the target circRNAs, etc. Targeting circRNAs and human diseases, in addition to miRNAs, is still in its infancy but important developments are expected.

\section{FUTURE PERSPECTIVES}

Interest in the contribution of circRNAs to the genesis and progression of human disorders is growing. While new information and insights into circular RNAs are generated rapidly, the biological and molecular mechanisms of circRNAs in the development of diverse diseases are not yet fully understood. These are likely to include miRNA sponging, splicing regulation and scaffolding for the assembly of macromolecular complexes (such as circRNA-protein complexes). In particular, little is known of the molecular mechanisms of their biogenesis, degradation and cellular localisation. It should be borne in mind that circRNAs may be a large family with widely diverse biogenesis, degradation, cellular localisation, function and functional mechanisms. Additional circRNAs will be identified as technology and research develops. Moreover, functional studies will disclose both physiological and pathological processes of the vast majority of circRNAs. We predict the construction of engineered circRNAs as molecular tools or treatments. Engineered circRNAs might be effective either for sequestering many RNAs and RNA-binding proteins or for releasing these stored molecules via cleavage of the circRNA.

CircRNAs provide new insights into the 'dark matter' of the human genome. The research and application prospects for circRNAs in human disorders are promising. CircRNAs may affect life processes, serve as diagnostic or predictive biomarkers of disease and provide new potential therapeutic targets. Our hope is that chemical and biotechnological advances will take place alongside basic studies, disclosing the physiological and pathological functions of circRNAs and developing circRNA-based therapeutic strategies, allowing safe and successful inclusion in day-to-day clinical practice.

Acknowledgements The authors apologise to those whose work has not been cited owing to limitations of space.

Contributors $\mathrm{C}-\mathrm{YH}$ and $\mathrm{L}-\mathrm{C}$ contributed equally to this paper. $\mathrm{C}-\mathrm{YH}, \mathrm{T}-\mathrm{CL}$ and $\mathrm{L}-\mathrm{XB}$ conceived the idea and collected literature; $\mathrm{C}-\mathrm{YH}$ and $\mathrm{L}-\mathrm{C}$ read and analysed the relevant literature; C-YH, L-C and T-CL wrote the manuscript; L-XB revised it.

Funding This work was supported, in part, by research grants from the China Postdoctoral Science Foundation (2015M582556), the Science and Technology Support Project of Sichuan Province (2015SZ0129; 2014SZ0002-6) and the Application Infrastructure Projects of Sichuan Province (2014YJ0180).

Competing interests None declared.

Provenance and peer review Not commissioned; externally peer reviewed.

\section{REFERENCES}

1 Memczak S, Jens M, Elefsinioti A, Torti F, Krueger J, Rybak A, Maier L, Mackowiak SD, Gregersen LH, Munschauer M, Loewer A, Ziebold U, Landthaler M, Kocks C, le Noble F, Rajewsky N. Circular RNAs are a large class of animal RNAs with regulatory potency. Nature 2013;495:333-8

2 Chen L, Huang C, Wang XL, Shan G. Circular RNAs in eukaryotic cells. Curr Genomics 2015;16:312-18.

3 Jeck WR, Sharpless NE. Detecting and characterizing circular RNAs. Nat Biotechnol 2014;32:453-61.

4 Li JQ, Yang J, Zhou P, Le YP, Zhou CW, Wang SM, Xu DZ, Lin HK, Gong ZH. Circular RNAs in cancer: novel insights into origins, properties, functions and implications. Am J Cancer Res 2015;5:472-80.

5 Peng L, Yuan XQ, Li GC. The emerging landscape of circular RNA ciRS-7 in cancer (review). Oncol Rep 2015;33:2669-74.
6 Zhao ZJ, Shen J. Circular RNA participates in the carcinogenesis and the malignant behavior of cancer. RNA Biol 2015: doi:10.1080/15476286.2015.1122162

7 Suzuki H, Tsukahara T. A view of pre-mRNA splicing from RNase R resistant RNAs. Int J Mol Sci 2014;15:9331-42.

8 Li Z, Huang C, Bao C, Chen L, Lin M, Wang X, Zhong G, Yu B, Hu W, Dai L, Zhu P, Chang Z, Wu Q, Zhao Y, Jia Y, Xu P, Liu H, Shan G. Exon-intron circular RNAs regulate transcription in the nucleus. Nat Struct Mol Biol 2015;22:256-64.

9 Wang Z. Not just a sponge: new functions of circular RNAs discovered. Sci China Life Sci 2015;58:407-8.

10 Ghosal S, Das S, Sen R, Basak P, Chakrabarti J. Circ2Traits: a comprehensive database for circular RNA potentially associated with disease and traits. Front Genet 2013:4:283.

11 Chen Z, Liu K, Yan Z, Xiang S, Sun Z. nc2Cancer: a database for cancer-associated human ncRNAs. Chin J Bioinformatics 2015;13:77-81 (in Chinese with English abstract).

12 Glažar P, Papavasileiou P, Rajewsky N. circBase: a database for circular RNAs. RNA 2014:20:1666-70.

13 Li JH, Liu S, Zhou H, Qu LH, Yang JH. starBase v2.0: decoding miRNA-ceRNA, miRNA-ncRNA and protein-RNA interaction networks from large-scale CLIP-Seq data. Nucleic Acids Res 2014;42:D92-7.

14 Liu YC, Li JR, Sun CH, Andrews E, Chao RF, Lin FM, Weng SL, Hsu SD, Huang CC, Cheng C, Liu CC, Huang HD. CircNet: a database of circular RNAs derived from transcriptome sequencing data. Nucleic Acids Res 2016;44:D209-15.

15 Zheng LL, Li JH, Wu J, Sun WJ, Liu S, Wang ZL, Zhou H, Yang JH, Qu LH. deepBase v2.0: identification, expression, evolution and function of small RNAs, LncRNAs and circular RNAs from deep-sequencing data. Nucleic Acids Res 2016;44: D196-202.

16 Dudekula DB, Panda AC, Grammatikakis I, De S, Abdelmohsen K, Gorospe M. Circlnteractome: a web tool for exploring circular RNAs and their interacting proteins and microRNAs. RNA Biol 2016;13:34-42.

17 Kozomara A, Griffiths-Jones S. miRBase: annotating high confidence microRNAs using deep sequencing data. Nucleic Acids Res 2014;42:D68-73.

18 Mokrejs M, Masek T, Vopálensky V, Hlubucek P, Delbos P, Pospisek M. IRESite-a tool for the examination of viral and cellular internal ribosome entry sites. Nucleic Acids Res 2010;38:D131-6.

19 Hansen TB, Jensen TI, Clausen BH, Bramsen JB, Finsen B, Damgaard CK, Kjems J. Natural RNA circles function as efficient microRNA sponges. Nature 2013;495:384-8.

20 Bhattacharya A, Cui Y. SomamiR 2.0: a database of cancer somatic mutations altering microRNA-ceRNA interactions. Nucleic Acids Res 2016;44:D1005-10.

21 Wang PL, Bao Y, Yee MC, Barrett SP, Hogan GJ, Olsen MN, Dinneny JR, Brown PO, Salzman J. Circular RNA is expressed across the eukaryotic tree of life. PLOS ONE 2014;9:e90859.

22 Hansen TB, Kjems J, Damgaard CK. Circular RNA and miR-7 in cancer. Cancer Res 2013;73:5609-12.

23 Zhang N, Li X, Wu CW, Dong Y, Cai M, Mok MTS, Wang H, Chen J, Ng SSM, Chen $M$, Sung JJY, Yu J. microRNA-7 is a novel inhibitor of YY1 contributing to colorectal tumorigenesis. Oncogene 2013:32:5078-88.

24 Kong X, Li G, Yuan Y, He Y, Wu X, Zhang W, Wu Z, Chen T, Wu W, Lobie P, Zhu T. MicroRNA-7 inhibits epithelial-to-mesenchymal transition and metastasis of breast cancer cells via targeting FAK expression. PLOS ONE 2012;7:e41523.

25 Lukiw WJ. Circular RNA (circRNA) in Alzheimer's disease (AD). Front Genet 2013:4:307.

26 Huang G, Zhu H, Shi Y, Wu W, Cai H, Chen X. cir-ITCH plays an inhibitory role in colorectal cancer by regulating the Wnt/beta-catenin pathway. PLOS ONE 2015;10: e0131225.

27 Li F, Zhang LY, Li W, Deng JQ, Zheng J, An MX, Lu JC, Zhou YF. Circular RNA ITCH has inhibitory effect on ESCC by suppressing the Wnt/beta-catenin pathway. Oncotarget 2015:6:6001-13.

28 Bachmayr-Heyda A, Reiner AT, Auer K, Sukhbaatar N, Aust S, Bachleitner-Hofmann T, Mesteri I, Grunt TW, Zeillinger R, Pils D. Correlation of circular RNA abundance with proliferation-exemplified with colorectal and ovarian cancer, idiopathic lung fibrosis and normal human tissues. Sci Rep 2015;5:8057

29 Li Y, Zheng Q, Bao C, Li S, Guo W, Zhao J, Chen D, Gu J, He X, Huang S. Circular RNA is enriched and stable in exosomes: a promising biomarker for cancer diagnosis. Cell Res 2015:25:981-4.

30 Qin M, Liu G, Huo X, Tao X, Sun X, Ge Z, Yang J, Fan J, Liu L, Qin W. Hsa_circ_0001649: a circular RNA and potential novel biomarker for hepatocellular carcinoma. Cancer Biomark 2016;16:161-9.

31 Li PF, Chen SC, Chen HL, Mo XY, Li TW, Shao YF, Xiao BX, Guo JM. Using circular RNA as a novel type of biomarker in the screening of gastric cancer. Clin Chim Acta 2015:444:132-6.

32 Wang YH, Yu XH, Luo SS, Han H. Comprehensive circular RNA profiling reveals that circular RNA100783 is involved in chronic CD28-associated CD8(+)T cell ageing. Immun Ageing 2015;12:17.

33 Qu S, Song W, Yang X, Wang J, Zhang R, Zhang Z, Zhang H, Li H. Microarray expression profile of circular RNAs in human pancreatic ductal adenocarcinoma. Genom Data 2015;5:385-7. 
34 Humphries B, Yang C. The microRNA-200 family: small molecules with novel roles in cancer development, progression and therapy. Oncotarget 2015;6:6472-98.

35 Esquela-Kerscher A, Slack FJ. Oncomirs-microRNAs with a role in cancer. Nat Rev Cancer 2006;6:259-69.

36 Garzon R, Calin GA, Croce CM. MicroRNAs in cancer. Annu Rev Med 2009;60:167-79.

37 Xiao-Jie L, Ai-Mei G, Li-Juan J, Jiang X. Pseudogene in cancer: real functions and promising signature. J Med Genet 2015;52:17-24.

38 Zhao X, Dou W, He L, Liang S, Tie J, Liu C, Li T, Lu Y, Mo P, Shi Y, Wu K, Nie Y, Fan D. MicroRNA-7 functions as an anti-metastatic microRNA in gastric cancer by targeting insulin-like growth factor-1 receptor. Oncogene 2013;32:1363-72.

39 Conn SJ, Pillman KA, Toubia J, Conn VM, Salmanidis M, Phillips CA, Roslan S, Schreiber AW, Gregory PA, Goodall GJ. The RNA binding protein quaking regulates formation of circRNAs. Cell 2015;160:1125-34.

40 Westholm JO, Miura P, Olson S, Shenker S, Joseph B, Sanfilippo P, Celniker SE, Graveley BR, Lai EC. Genome-wide analysis of drosophila circular RNAs reveals their structural and sequence properties and age-dependent neural accumulation. Cell Rep 2014;9:1966-80.

41 Ivanov A, Memczak S, Wyler E, Torti F, Porath HT, Orejuela MR, Piechotta M, Levanon EY, Landthaler M, Dieterich C, Rajewsky N. Analysis of intron sequences reveals hallmarks of circular RNA biogenesis in animals. Cell Rep 2015;10:170-7.

42 Rybak-Wolf A, Stottmeister C, Glažar P, Jens M, Pino N, Giusti S, Hanan M, Behm M, Bartok O, Ashwal-Fluss R, Herzog M, Schreyer L, Papavasileiou P, Ivanov A, Ohman $M$, Refojo D, Kadener $S$, Rajewsky N. Circular RNAs in the mammalian brain are highly abundant, conserved and dynamically expressed. Mol Cell 2015;58:870-85.

43 You X, Vlatkovic I, Babic A, Will T, Epstein I, Tushev G, Akbalik G, Wang M, Glock C, Quedenau C, Wang X, Hou J, Liu H, Sun W, Sambandan S, Chen T, Schuman EM, Chen W. Neural circular RNAs are derived from synaptic genes and regulated by development and plasticity. Nat Neurosci 2015;18:603-10.

44 Hansen TB, Wiklund ED, Bramsen JB, Villadsen SB, Statham AL, Clark SJ, Kjems J. miRNA-dependent gene silencing involving Ago2-mediated cleavage of a circular antisense RNA. EMBO J 2011:30:4414-22.

45 Armakola M, Higgins MJ, Figley MD, Barmada SJ, Scarborough EA, Diaz Z, Fang $X$ Shorter J, Krogan NJ, Finkbeiner S, Farese RV Jr, Gitler AD. Inhibition of RNA lariat debranching enzyme suppresses TDP-43 toxicity in ALS disease models. Nat Genet 2012;44:1302-9.
46 Burd CE, Jeck WR, Liu Y, Sanoff HK, Wang Z, Sharpless NE. Expression of linear and novel circular forms of an INK4/ARF-associated non-coding RNA correlates with atherosclerosis risk. PLoS Genet 2010;6:e1001233.

47 Holdt LM, Beutner F, Scholz M, Gielen S, Gabel G, Bergert H, Schuler G, Thiery J, Teupser D. ANRIL expression is associated with atherosclerosis risk at chromosome 9p21. Arterioscler Thromb Vasc Biol 2010;30:620-7.

48 Holdt LM, Hoffmann S, Sass K, Langenberger D, Scholz M, Krohn K, Finstermeier K, Stahringer A, Wilfert W, Beutner F, Gielen S, Schuler G, Gabel G, Bergert H, Bechmann I, Stadler PF, Thiery J, Teupser D. Alu elements in ANRIL non-coding RNA at chromosome 9p21 modulate atherogenic cell functions through trans-regulation of gene networks. PLoS Genet 2013;9:e1003588.

49 Salzman J, Chen RE, Olsen MN, Wang PL, Brown PO. Cell-type specific features of circular RNA expression. PLoS Genet 2013;9:e1003777.

50 Ashwal-Fluss R, Meyer M, Pamudurti NR, Ivanov A, Bartok O, Hanan M, Evantal N, Memczak S, Rajewsky N, Kadener $\mathrm{S}$. circRNA biogenesis competes with pre-mRNA splicing. Mol Cell 2014;56:55-66.

51 Lasda E, Parker R. Circular RNAs: diversity of form and function. RNA 2014;20:1829-42.

52 Shen T, Han M, Wei G, Ni T. An intriguing RNA species-perspectives of circularized RNA. Protein Cell 2015;6:871-80.

53 Chen I, Chen CY, Chuang TJ. Biogenesis, identification and function of exonic circular RNAs. Wiley Interdiscip Rev RNA 2015;6:563-79.

54 Memczak S, Papavasileiou P, Peters 0, Rajewsky N. Identification and characterization of circular RNAs as a new class of putative biomarkers in human blood. PLOS ONE 2015;10:e0141214.

55 Lin X, Lo HC, Wong DT, Xiao X. Noncoding RNAs in human saliva as potential disease biomarkers. Front Genet 2015;6:175.

56 Bahn JH, Zhang Q, Li F, Chan TM, Lin X, Kim Y, Wong DTW, Xiao X. The landscape of MicroRNA, Piwi-Interacting RNA and circular RNA in human saliva. Clin Chem 2015;61:221-30.

57 Liu YC, Cui HZ, Wang WJ, Li LN, Wang ZX, Yang S, Zhang XJ. Construction of circular miRNA sponges targeting miR-21 or miR-221 and demonstration of their excellent anticancer effects on malignant melanoma cells. Int J Biochem Cell B 2013:45:2643-50.

58 Kosik KS. Molecular biology: circles reshape the RNA world. Nature 2013;495:322-4. 